\title{
Rapamycin Inhibitors for Eye Squamous Cell Carcinoma after Renal Transplantation: A Case Report
}

\author{
Barbara Infante $^{a}$ Nicola Coviello ${ }^{a}$ Dario Troise $^{a}$ Matteo Gravina ${ }^{b}$ \\ Valeria Bux c Giuseppe Castellano ${ }^{a}$ Giovanni Stallone ${ }^{a}$ \\ ${ }^{a}$ Department of Medical and Surgical Sciences, Nephrology, Dialysis and Transplantation Unit, University of \\ Foggia, Foggia, Italy; 'bepartment of Medical and Surgical Sciences, Radiology Unit, University of Foggia, Foggia, \\ Italy; 'Department of Medical and Surgical Sciences, Ophthalmology Unit, University of Foggia, Foggia, Italy
}

\section{Keywords}

Rapamycin inhibitors · Squamous cell carcinoma · Eye · Kidney transplant

\begin{abstract}
Introduction: The immunosuppressive efficiency obtained in the last decades in kidney transplantation significantly improved graft survival. However, there is still a high risk and incidence of cancer in transplant patients strongly and directly related to the type of immunosuppression. An increasing body of evidence suggests that the PI3K/Akt/mTOR pathway may play a pivotal role in the development and progression of several neoplastic diseases. Case Presentation: We describe a 47-year-old male patient who received a cadaveric primary renal transplant in November 2008 developing a poorly differentiated infiltrating and ulcerated squamous cell carcinoma (SCC) at the eye level. In this patient, the modification of an immunosuppressive regimen with introduction of rapamycin (mTOR) inhibitors and withdrawal of calcineurin inhibitors (CNIs) led to the resolution of this severe condition. Conclusion: The introduction of mTOR inhibitors and withdrawal of CNIs in kidney-transplanted patients with de novo eye SCC should be considered in this clinical setting.

(c) 2021 The Author(s)

Published by S. Karger AG, Basel
\end{abstract}

\section{Introduction}

The immunosuppressive efficiency obtained in the last decades in kidney transplantation significantly improved graft survival [1]. In fact, the incidence of acute rejection has significantly decreased due to the use of more potent and specific immunosuppressive drugs [2]. On the other hand, the incidence and prevalence of cancer in transplant patients have increased in the last $10-15$ years partly because of higher patient/donor age and the use of new immunosuppressive agents [3]. The high risk and incidence of cancer in transplant patients are directly related to the type of immunosuppressive therapy, its duration, doses, and blood levels [4]. Interestingly, the types of cancer developed by the transplant population differ from those in the general population, which can partly be explained by the distinct course of the oncoviruses developed in transplant patients, especially human papillomavirus, Epstein-Barr virus, and human herpes simplex-8 [5]. In addition, the prevalent posttransplantation malignancy of a specific organ also varies because of the different environmental exposure.

Calcineurin inhibitor (CNI)-based regimen is the main immunosuppressive protocol used in renal transplantation $[5,6]$. CNIs, by impairing the nucleotide excision repair and activation of the tumor suppression karger@karger.com www.karger.com/kbr

Karger $\stackrel{\text { ' }}{5}$

GOPEN ACCESS
(C) 2021 The Author(s)

Published by S. Karger AG, Basel

This article is licensed under the Creative Commons AttributionNonCommercial-NoDerivatives 4.0 International License (CC BYNC-ND) (http://www.karger.com/Services/OpenAccessLicense). Usage and distribution for commercial purposes as well as any distribution of modified material requires written permission.
Giovanni Stallone

Department of Medical/Surgical Sciences, Nephrology

Dialysis and Transplantation Unit, University of Foggia

Viale Luigi Pinto, 1, IT-71100 Foggia (Italy)

giovanni.stallone@ unifg.it 
gene, are commonly mentioned as tumor initiators in several studies $[7,8]$. The mammalian target of the rapamycin (mTOR) pathway plays an important role in the development of distinct kinds of tumor. mTOR, a cytoplasmic serine-threonine kinase, is a key molecular switch of protein translation and cell cycle progression [9]. An increasing body of evidence suggests that the $\mathrm{PI} 3 \mathrm{~K} / \mathrm{Akt} / \mathrm{mTOR}$ pathway may play a pivotal role in the development and progression of several neoplastic diseases [10-12]. mTOR sits at an intersection of several inputs to the cell, integrating signals from both growth factors and nutrients and mediating its effects through 2 main effectors, p70S6 kinase and 4E-BP1/eIF4E, with a well-defined role in the regulation of protein translation [11-13]. The mTOR pathway can, indeed, promote neoplastic cell survival and proliferation through the phosphorylation and sequestration of the proapoptotic Bad [14], upregulation of FLIPs [15], and translation of antiapoptotic proteins, supporting the mitochondrial energy production $[11,16,17]$. Thus, great interest has arisen in the potential benefits of mTOR inhibition in tumors $[12,13]$.

\section{Case Report}

We describe a 47-year-old male patient who received a cadaveric primary renal transplant in November 2008. An immunosuppressive therapy regimen was based on CNIs (tacrolimus at a dosage of $0.01 \mathrm{mg} / \mathrm{kg} / \mathrm{die}$ ) and corticosteroids. Since transplantation, he began routine follow-ups at our medical division, with good clinical condition and stable graft function (serum $\mathrm{Cr} 0.9 \mathrm{mg} / \mathrm{dL}$ ) without urinary abnormalities (Table 1).

In August 2017, the patient referred the first appearance of conjunctival inflammation with severe tearing, followed by the development of a cutaneous lesion at the left eye caruncle, without vision alterations; the patient had a stable renal function. Following ophthalmological evaluation, a local corticosteroid and antibiotic therapy was attempted without benefits. Therefore, we started to hypothesize the presence of a cancer lesion; the patient underwent an excisional cutaneous biopsy of periocular lesion (Fig. 1a) with subsequent diagnosis of poorly differentiated infiltrating and ulcerated squamous cell carcinoma (SCC); the borders of the lesion were analyzed, resulting disease-free (Fig. 1c). To complete the diagnosis, a brain magnetic resonance imaging (MRI) was performed with discovery, near to the left eye caruncle, of a "dishomogeneous neoformation with contrast enhancement without a clear-cut separation from the adjacent muscle and bones systems" (Fig. 2a).

Our first therapeutic approach was to reduce CNI dosage $(0.005 \mathrm{mg} / \mathrm{kg} / \mathrm{die})$ starting with an mTOR inhibitor everolimus at an initial dose of $1.5 \mathrm{mg} /$ day $(0.75 \mathrm{mg}$ bid). The patient continued weekly ambulatory eye medications presenting stable graft function.

Six months after eye biopsy, a new brain magnetic resonance imaging was repeated revealing the presence of an "enhancement
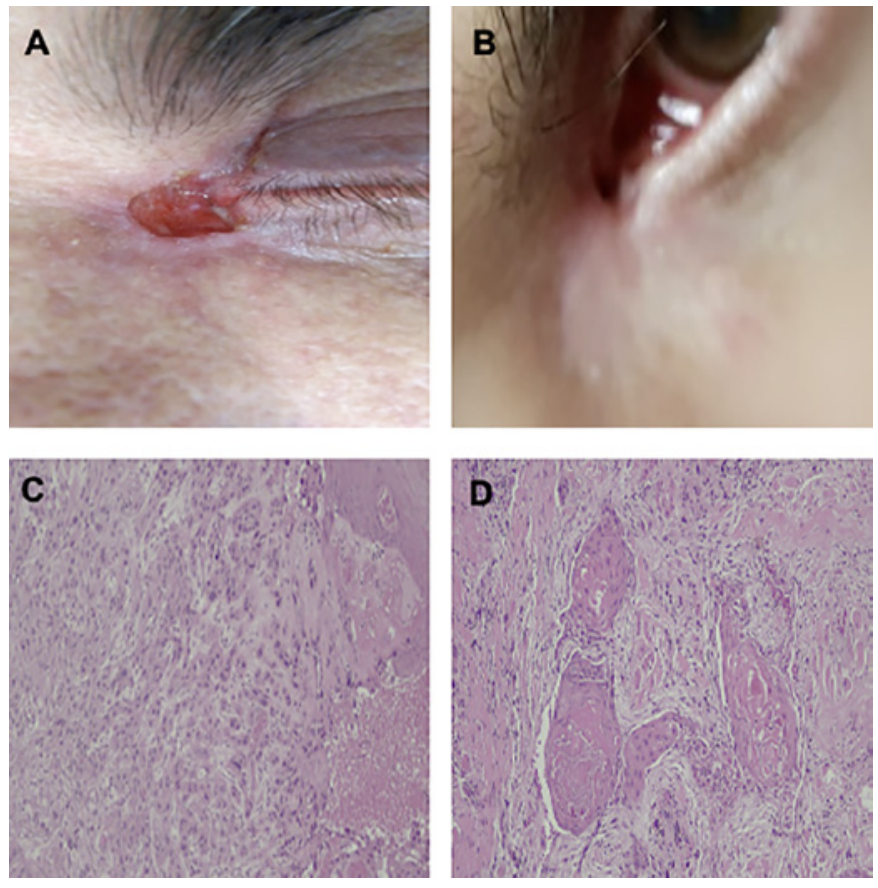

Fig. 1. A Cutaneous lesion at the left eye caruncle after excisional cutaneous biopsy. B Ocular lesion 1 year after CNI withdrawn and mTORi introduced as unique immunosuppressive agent. As showed, there was a marked improvement of the ocular lesion. C Poorly differentiated SCC with infiltrative ulcerated anaplastic behavior. Earlier, the lesion margins were not free of disease (hematoxylin and eosin staining). D Moderately differentiated SCC, with muscle and connective tissues infiltrating (hematoxylin and eosin staining). CNI, calcineurin inhibitor; mTOR, rapamycin; SCC, squamous cell carcinoma.

contrast region in previous surgical resection place" (Fig. 2b). Suspecting disease diffusion, a second biopsy was done, showing skin cancer infiltration on subcutaneous and muscular tissues (Fig. 1d). Since the unfavorable prognosis with a histological result, the ophthalmologist recommended the surgical removal of the whole orbit. The patient was informed, and he refused surgery; considering the stability of renal function and the absence of proteinuria, $\mathrm{CNI}$ was withdrawn and everolimus was adopted as a single immunosuppressive drug. We administered the drug at an initial dose of $2 \mathrm{mg} /$ day (1 mg bid) to obtain a blood trough level of 3-5 $\mathrm{ng} / \mathrm{mL}$.

One year later, the patient's clinical condition was stable (Fig. 1b); a new radiological control was performed, demonstrating no contrast enhancement areas in the previous cancer region and a complete remission of the original lesion (Fig. 2c).

Actually, the patient continued everolimus at low doses $(1 \mathrm{mg}$ day: $0.5 \mathrm{mg}$ bid, obtaining a mean blood trough level of $3.4 \pm 0.7$ $\mathrm{ng} / \mathrm{mL}$ ), with a good renal allograft function and absence of proteinuria. During the entire follow-up period, mTORi were well tolerated, and the patient did not present side effects (Table 1). The patient gave written informed consent to publish this case report, including publication of the images. 
Table 1. Clinical and demographic data of the patient

\begin{tabular}{|c|c|c|c|c|}
\hline \multicolumn{2}{|l|}{ Age at dialysis, yrs } & \multicolumn{3}{|l|}{37} \\
\hline \multicolumn{2}{|l|}{ ESRD etiology } & \multicolumn{3}{|l|}{ Unknown } \\
\hline \multicolumn{2}{|l|}{ Dialytic treatment } & \multicolumn{3}{|l|}{ CAPD } \\
\hline \multicolumn{2}{|l|}{ Age at transplant, yrs } & \multicolumn{3}{|l|}{38} \\
\hline \multicolumn{2}{|l|}{ Age at SCC diagnosis, yrs } & \multicolumn{3}{|l|}{47} \\
\hline \multicolumn{2}{|c|}{ Immunosuppressive therapy at transplant } & \multicolumn{3}{|l|}{ CS, Tac } \\
\hline \multicolumn{2}{|c|}{ Immunosuppressive therapy at SCC diagnosis } & \multicolumn{3}{|c|}{ CS, Tac (low dose), mTORi (low dose) } \\
\hline \multicolumn{2}{|c|}{ Immunosuppressive therapy 1 yr post-SCC diagnosis } & \multicolumn{3}{|c|}{ CS, mTORi } \\
\hline \multicolumn{2}{|l|}{ Acute rejection episodes } & \multicolumn{3}{|l|}{ None } \\
\hline Chemistry & $\begin{array}{l}\text { Transplant } \\
\text { center discharge }\end{array}$ & SCC diagnosis & $\mathrm{CNI}+\mathrm{mTORi}$ & mTORi \\
\hline $\mathrm{sCr}, \mathrm{mg} / \mathrm{dL}$ & 2.4 & 1.02 & 0.9 & 0.95 \\
\hline eGFR MDRD, $\mathrm{mL} / \mathrm{min} / 1.73 \mathrm{~m}^{2}$ & 32.4 & 83.2 & 96 & 92 \\
\hline Proteinuria, $\mathrm{g} / 24 \mathrm{~h}$ & 0.57 & 0.6 & 0.29 & 0.62 \\
\hline Fasting glucose, $\mathrm{mg} / \mathrm{dL}$ & 98 & 106 & 101 & 82 \\
\hline Total cholesterol, mg/dL & 200 & 216 & 237 & 244 \\
\hline Triglycerides, mg/dL & 98 & 232 & 258 & 203 \\
\hline
\end{tabular}

CNI, calcineurin inhibitor; mTOR, rapamycin; SCC, squamous cell carcinoma.

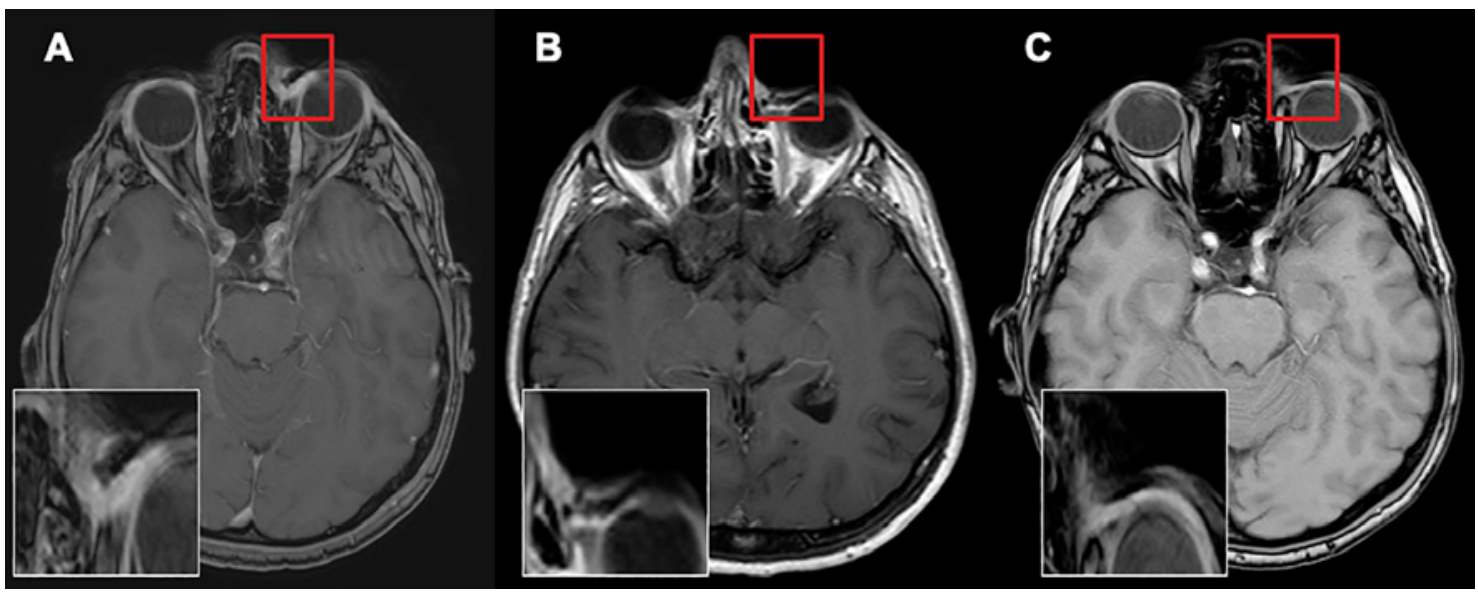

Fig. 2. A Radiological features of the first brain MRI performed for diagnostic completion. It shows the presence near the left eye caruncle, of a "dishomogeneous neoformation with contrast enhancement (overintense signal at MRI imaging) without a clearcut separation from the adjacent muscle and bones systems" (red color line highlighted in the bottom panel). B Radiological features of the new brain MRI performed 6 months after mTORi introduction with CNI reduction. It reveals the presence of an "enhancement contrast region in previous surgical resection place" and

\section{Discussion}

SCC of the eye occurs at substantially increased rates in individuals with iatrogenic or congenital immunodeficiency or in those affected by human immunodeficiency shows a disappearance of previous overintense signal with a minimal residual area near medial orbital section (red color line highlighted in the bottom panel) due to neoplastic infiltration. C Radiological features of the new brain MRI performed 1 year after CNI withdrawn and mTORi introduction. It shows no contrast enhancement areas in the previous cancer region and a complete remission of the original lesion (red color line highlighted in the bottom panel). CNI, calcineurin inhibitor; mTOR, rapamycin; MRI, magnetic resonance imaging.

virus (HIV). The rates of this cancer in transplant patients are approximately 20 -fold compared to the general population, while in individuals with HIV infection, the rate is 13 times higher [18]. Moreover, while the increased risk of ocular SCC in HIV infection appears to be associated 
with exposure to high levels of solar ultraviolet radiation [19] and infection with high-risk human papillomavirus subtypes [20,21], and in renal transplant patients, this risk is strongly associated with immunosuppressive drugs used to prevent graft rejection. The fact that the development of this cancer is associated with the cumulative immunosuppression dose was validated by registry data which showed that all patients who developed SCC had a history of glomerulonephritis treated with immunosuppressive agents before transplantation [18].

SCC of the eye is almost uniformly the result of local invasion from a cutaneous primary lesion, extension by perineural invasion, or metastasis. This particular form of carcinoma for localization and diffusion can be compared to a rare form of SCC as primary orbital SCC is an extremely rare disease without a specific treatment. This was evident in case reports described in the literature where the management ranged from primary orbital radiation to orbital exenteration with adjuvant chemotherapy and orbital radiation [22]. In fact, in our case, the first indication of the ophthalmologist was the surgical removal of whole orbit, followed by adjuvant chemotherapy and orbital radiation with a high probability of recurrence. Then, considering the patient's decision, our positive experience in the use of mTORi in the setting of kidney transplant patients affected by cancer, and the existing literature [23-25], CNI was withdrawn, and the patient continued with mTORi as the only antirejection drug.

Although the onset of neoplastic diseases in renal transplant patients may be related to the increased occurrence of viral infections or the direct effect of immunosuppressive drugs on specific cell populations, these causes alone cannot justify the high incidence of malignancies in this setting $[26,27]$. On the other hand, the role of the immune system in controlling tumor development and progression is widely accepted and might be even more relevant in the transplant scenario. For these reasons, disease-specific survival is significantly worse among transplant recipients with solid cancer compared to nontransplant patients with the same malignancies [28].

Immunosuppression is considered the most important risk factor as it decreases the immunologic control of oncogenic viral infection and cancer immunosurveillance. Although it is accepted that the overall immunosuppressive dose is associated with the increased cancer risk following transplantation, the relative effect of different immunosuppressive agents is not well established at this time.
Several studies demonstrated that apart from the effect on IL-2 expression, CNI exerts an array of effects potentially promoting the development and progression of neoplastic diseases including transforming growth factor $\beta 1$ production and suppression of antitumor-specific immune responses $[29,30]$. In addition, CNIs induce the expression of vascular endothelial growth factor, leading to an increased tumor angiogenesis [31] by inhibiting cancer cell apoptosis, through a calcineurin-dependent pathway $[32,33]$. It is noteworthy that the pro-neoplastic effects of CNIs were counterbalanced by the simultaneous administration of mTORi in different experimental models [34, 35], and data registries demonstrated that maintenance of mTORi-based immunosuppression is characterized by a significantly reduced risk to develop any de novo posttransplant malignancy or nonskin solid malignancy compared to a CNI-based immunosuppressive regimen [36]. The cancer-promoting effect of CNIs, independent of reduced immunosurveillance, has been clearly shown and currently mTORi is the only class of immunosuppressive drugs that have been shown to exert simultaneously immunosuppressive and antineoplastic effects [37].

\section{Conclusions}

At our knowledge, this is the first case of de novo eye SCC treated with mTORi; however, it is important to realize that our approach might not be used in all cases of eye SCC. Effectively, our case suggests that patients who develop posttransplant malignancies represent a serious challenge for transplant physicians. The management of immunosuppressive therapy in this setting is still debated. Although evidence-based guidelines are missing, the decision should consider the type and stage of malignancies along with actual graft function. Withdrawal of CNI and introduction of mTORi are fully supported by the existing literature. However, the use of mTORi in patients with posttransplant malignancies, instead of simply withdrawing immunosuppressive therapy, can be considered as an option to preserve graft function and, at the same time, to reduce the effect of immunosuppression on neoplastic disease progression.

\section{Statement of Ethics}

This research was carried out in accordance with the guidelines of the World Medical Association Declaration of Helsinki. The patient gave written informed consent to publish this case, including publication of images. 


\section{Conflict of Interest Statement}

The authors declare no conflict of interest.

\section{Funding Sources}

The paper was not supported by any founding source.

\section{Author Contributions}

I.B.: analyzed data and wrote the manuscript. C.N.: followed up the patient and collected data. T.D.: followed up the patient and collected data. G.M.: performed radiological examinations and analyzed data. B.V.: followed up the patient and collected data. C.G.: analyzed data and wrote the manuscript. S.G.: interpreted data and revised the manuscript.

\section{References}

1 Wong G, Chapman JR. Cancers after renal transplantation. Transplant Rev. 2008;22(2): 141.

2 Chang SH, Russ GR, Chadban SJ, Campbell SB, McDonald SP. Trends in kidney transplantation in Australia and New Zealand, 19932004. Transplantation. 2007;84(5):611.

3 Webster AC, Craig JC, Simpson JM, Jones MP, Chapman JR. Identifying high risk groups and quantifying absolute risk of cancer after kidney transplantation: a cohort study of 15,183 recipients. Am J Transplant. 2007;7(9):2140.

4 Tremblay F, Fernandes M, Habbab F, deB Edwardes MD, Loertscher R, Meterissian S. Malignancy after renal transplantation: incidence and role of type of immunosuppression. Ann Surg Oncol. 2002;9(8):785.

5 Vanhove T, Annaert P, Kuypers DR. Clinical determinants of calcineurin inhibitor disposition: a mechanistic review. Drug Metab Rev. 2016;48(1):88-112.

6 Malvezzi P, Jouve T, Rostaing L. Costimulation blockade in kidney transplantation: an update. Transplantation. 2016;100(11):2315-23.

7 Euvrard S, Kanitakis J, Claudy A. Skin cancers after organ transplantation. N Engl J Med. 2003;348(17):1681-91.

8 Yarosh DB, Pena AV, Nay SL, Canning MT, Brown DA. Calcineurin inhibitors decrease DNA repair and apoptosis in human keratinocytes following ultraviolet B irradiation. J Invest Dermatol. 2005;125(5):1020-5.

9 Bjornsti MA, Houghton PJ. The TOR pathway: a target for cancer therapy. Nat Rev Cancer. 2004;4(5):335-48.

10 Wendel HG, De Stanchina E, Fridman JS, Malina A, Ray S, Kogan S, et al. Survival signalling by Akt and eIF4E in oncogenesis and cancer therapy. Nature. 2004;428(6980):332-7.

11 Guertin DA, Sabatini DM. Defining the role of mTOR in cancer. Cancer Cell. 2007;12(1):922.

12 Chiang GG, Abraham RT. Targeting the mTOR signaling network in cancer. Trends Mol Med. 2007;13(10):433-42.

13 Abraham RT, Gibbons JJ. The mammalian target of rapamycin signalling pathway: twists and turns in the road to cancer therapy. Clin Cancer Res. 2007;13:3109-14.

14 Harada H, Andersen JS, Mann M, Terada N, Korsmeyer SJ. p70S6 kinase signals cell survival as well as growth, inactivating the pro-apoptotic molecule BAD. Proc Natl Acad Sci U S A. 2001;98(17):9666-70.
15 Panner A, James CD, Berger MS, Pieper RO. mTOR controls FLIPs translation and TRAIL sensitivity in glioblastoma multiforme cells. Mol Cell Biol. 2005;25(20):8809-23.

16 Edinger AL, Thompson CB. An activated mTOR mutant supports growth factor-independent, nutrient-dependent cell survival. Oncogene. 2004;23(33):5654-63.

17 Weinstein IB, Joe AK. Mechanisms of disease: oncogene addiction a rationale for molecular targeting in cancer therapy. Nat Clin Pract Oncol. 2006;3:448-57.

18 Vajdic CM, van Leeuwen MT, McDonald SP, McCredie RE, Law M, Chapmen JR, et al. Increased incidence of squamous cell carcinoma of eye after kidney transplantation. J Natl Cancer Inst. 2007;99:1340-2.

19 Mbulaiteye SM, Katabira ET, Wabinga H, Parkin DM, Virgo P, Ochai R, et al. Spectrum of cancers among HIV-infected persons in Africa: the Uganda AIDS-Cancer Registry Match Study. Int J Cancer. 2006;118(4):985-90.

20 Ateenyi-Agaba C, Dai M, Le Calvez F, Katongole-Mbidde E, Smet A, Tommasino M, et al. TP53 mutations in squamous-cell carcinomas of the conjunctiva: evidence for UV-induced mutagenesis. Mutagenesis. 2004;19(5):399401.

21 Ateenyi-Agaba C, Weiderpass E, Smet A, Dong W, Dai M, Kahwa B, et al. Epidermodysplasia verruciformis human papillomavirus types and carcinoma of the conjunctiva: a pilot study. Br J Cancer. 2004;90(9):1777-9.

22 Sun EC, Fears TR, Goedert JJ. Epidemiology of squamous cell conjunctival cancer. Cancer Epidemiol Biomarkers Prev. 1997;6(2):73-7.

23 Blandford AD, Bellerive C, Tom M, Koyfman S, Adelstein DJ, Plesec TP, et al. Case report: primary orbital squamous cell carcinoma. Ocul Oncol Pathol. 2019;5(1):60-5.

24 Stallone G, Schena A, Infante B, Di Paolo S, Loverre A, Maggio G, et al. Sirolimus for Kaposi's sarcoma in renal-transplant recipients. N Engl J Med. 2005;352(13):1317-23.

25 Stallone G, Infante B, Grandaliano G, Bristogiannis G, Macarini L, Mezzopane D, et al. Rapamycin for treatment of type I autosomal dominant polycystic kidney disease [RAPYDstudy]: a randomized, controlled study. Nephrol Dial Transplant. 2012;27:3560-7.

26 Stallone G, Infante B, Grandaliano G. Management and prevention of post-transplant malignancies in kidney transplant recipients. Clin Kidney J. 2015;8(5):637-44.
27 Vajdic CM, van Leeuwen MT. Cancer incidence and risk factors after solid organ transplantation. Int J Cancer. 2009;125(8):1747-54.

28 Gruber SA, Gillingham K, Sothern RB, Stephanian E, Matas AJ, Dunn DL. De novo cancer in cyclosporine-treated and non-cyclosporinetreated adult primary renal allograft recipients. Clin Transplant. 1994;8(4):388-95.

29 Cherikh WS, Kauffman HM, McBride MA, Maghirang J, Swinnen LJ, Hanto DW. Association of the type of induction immunosuppression with posttransplant lymphoproliferative disorder, graft survival, and patient survival after primary kidney transplantation. Transplantation. 2003;76(9):1289-93.

30 Shihab FS, Andoh TF, Tanner AM, Noble NA, Border WA, Franceschini N, et al. Role of transforming growth factor-beta 1 in experimental chronic cyclosporine nephropathy. Kidney Int. 1996;49(4):1141-51.

31 Teicher BA. Malignant cells, directors of the malignant process: role of TGF $\beta$. Cancer Metastasis Rev. 2001;20:133-43.

32 Hojo M, Morimoto T, Maluccio M, Asano T, Morimoto K, Lagman M, et al. Cyclosporine induces cancer progression by a cell-autonomous mechanism. Nature. 1999;397(6719): $530-4$.

33 Rivera A, Maxwell SA. The p53-induced gene6 (proline oxidase) mediates apoptosis through a calcineurin-dependent pathway. J Biol Chem. 2005;280(32):29346-54.

34 Sugie N, Fujii N, Danno K. Cyclosporin-A suppresses p53-dependent repair DNA synthesis and apoptosis following ultraviolet-B irradiation. Photodermatol Photoimmunol Photomed. 2002;18(4):163-8.

35 Luan FL, Hojo M, Maluccio M, Yamaji K, Suthanthiran M. Rapamycin blocks tumor progression: unlinking immunosuppression from antitumor efficacy. Transplantation. 2002;73(10):1565-72.

36 Koehl GE, Andrassy J, Guba M, Richter S, Kroemer A, Scherer MN, et al. Rapamycin protects allografts from rejection while simultaneously attacking tumors in immunosuppressed mice. Transplantation. 2004;77(9):1319-26.

37 Yanik EL, Gustafson SK, Kasiske BL, Israni AK, Snyder JJ, Hess GP, et al. Sirolimus use and cancer incidence among US kidney transplant recipients. Am J Transplant. 2015;15(1):12936. 